\title{
Systematic study of the static electrical properties of the CO molecule: Influence of the basis set size and correlation energy
}

\author{
Josep M. Luis, Josep Martí, Miquel Duran, and José L. Andrés ${ }^{a)}$ \\ Institute of Computational Chemistry and Department of Chemistry, University of Girona, 17071 Girona, \\ Catalonia, Spain
}

(Received 7 December 1994; accepted 7 February 1995)

\begin{abstract}
The influence of the basis set size and the correlation energy in the static electrical properties of the CO molecule is assessed. In particular, we have studied both the nuclear relaxation and the vibrational contributions to the static molecular electrical properties, the vibrational Stark effect (VSE) and the vibrational intensity effect (VIE). From a mathematical point of view, when a static and uniform electric field is applied to a molecule, the energy of this system can be expressed in terms of a double power series with respect to the bond length and to the field strength. From the power series expansion of the potential energy, field-dependent expressions for the equilibrium geometry, for the potential energy and for the force constant are obtained. The nuclear relaxation and vibrational contributions to the molecular electrical properties are analyzed in terms of the derivatives of the electronic molecular properties. In general, the results presented show that accurate inclusion of the correlation energy and large basis sets are needed to calculate the molecular electrical properties and their derivatives with respect to either nuclear displacements or/and field strength. With respect to experimental data, the calculated power series coefficients are overestimated by the SCF, CISD, and QCISD methods. On the contrary, perturbation methods (MP2 and MP4) tend to underestimate them. In average and using the $6-311+\mathrm{G}(3 d f)$ basis set and for the $\mathrm{CO}$ molecule, the nuclear relaxation and the vibrational contributions to the molecular electrical properties amount to $11.7 \%, 3.3 \%$, and $69.7 \%$ of the purely electronic $\mu, \alpha$, and $\beta$ values, respectively. (C) 1995 American Institute of Physics.
\end{abstract}

\section{INTRODUCTION}

In the last years, there has been a growing interest for the nonlinear optical properties of polyatomic molecules. ${ }^{1-3}$ Such properties give the response of a molecule which is placed under the influence of an electromagnetic radiation. Under these conditions, and taking into account only the stronger electric field component, the potential energy of a molecule can be expanded in a Taylor series,

$$
\begin{aligned}
V= & V_{0}-\sum_{i}^{x, y, z} \mu_{i} F_{i}-\frac{1}{2 !} \sum_{i, j}^{x, y, z} \alpha_{i j} F_{i} F_{j} \\
& -\frac{1}{3 !} \sum_{i, j, k}^{x, y, z} \beta_{i j k} F_{i} F_{j} F_{k}-\cdots .
\end{aligned}
$$

If the molecular properties ( $P$ in general) are defined from the Taylor series of the dipole moment $\mu$, the linear response is given by the polarizability $\alpha$, and the nonlinear terms of the series are given by the $n$ th-order hyperpolarizabilities $(\beta$ and $\gamma)$. The dynamic properties are defined for timeoscillating fields, whereas static properties are obtained if the electric field strength is time-independent. In this study, only the static, space-uniform field has been considered, because it allows for the determination of static electrical properties.

When a molecule is placed under the effect of an electric field, the electronic cloud is modified, nuclei positions are changed and vibrational (and rotational) motion is

\footnotetext{
a) Author to whom correspondence should be addressed.
}

perturbed. ${ }^{4-12}$ All these changes can be explained in terms of the electrical properties, namely, dipole moment, polarizability, and $n$ th-order hyperpolarizabilities. Experimental information of such changes induced by the electric field can be obtained from the vibrational Stark effect (VSE) and the vibrational intensity effect (VIE). These effects are reported from the Stark tuning rate $\left(\delta_{\nu E}\right)$ and from the infrared cross section $\left(\delta_{S E}\right)$, respectively. ${ }^{13-16}$

The methodology employed in this paper, which can be extended to polyatomic molecules, will allow us to consider the most important contributions to the molecular properties. Although some studies ${ }^{12,14-16}$ have dealt with this subject earlier, a systematic study of those properties is still missing. In this work, the potential energy of a chemical system will be expanded in a double power series. Then, the effect of both mechanical and electrical anharmonicity corrections will be included. The purpose of this paper is, thus, to assess the importance of correlation energy, basis set size, and truncation in the power series. One must note that this method can also be related to the more traditional perturbation treatment. $^{17}$

For the molecular properties of the $\mathrm{CO}$ molecule, a fair amount of data, either theoretical calculations ${ }^{18-27}$ or experimental determinations, ${ }^{28-38}$ have been reported. To our knowledge, few studies have been reported referring to the molecular property derivatives. As it will be shown in Sec. II, the Stark tuning rate $\left(\delta_{\nu E}\right)$, the infrared cross section $\left(\delta_{S E}\right)$ and the nuclear relaxation $\left(P_{\mathrm{nr}}\right)$ and vibrational contri- 
butions $\left(P_{\text {vib }}\right)$ to the molecular properties are expressed in terms of these derivatives.

In Sec. II A, we present the details of the molecular orbital (MO) ab initio calculations carried out in this paper, and in Sec. II B, we report the relationships between $\delta_{\nu E}, \delta_{S E}$, $P_{\mathrm{nr}}, P_{\text {vib }}$ and the coefficients of the power series. The molecular property derivatives for different levels of theory will be presented in Sec. III. Then, the effect of the basis sets and correlation energy will be analyzed. From these coefficients, the $\delta_{\nu E}$, the $\delta_{S E}$ and the nuclear relaxation and vibrational contributions to dipole moment, polarizability, and first hyperpolarizability will be presented and compared with respect to available experimental data.

\section{METHODOLOGY}

\section{A. Details of the $a b$ initio calculations}

Calculations have been carried out at the $a b$ initio $\mathrm{MO}$ level of theory. Inclusion of correlation energy has been considered through perturbation theory at the MP2 (Ref. 39) and MP4 (Ref. 40) levels, and through the iterating methods CI (Ref. 41) and QCI (Ref. 42) including all singles and doubles excitations. The basis sets used in this work are the splitvalence $3-21 G,{ }^{43}$ the split-valence including diffuse functions $6-31+\mathrm{G},{ }^{44,45}$ polarization functions $6-31 \mathrm{G}(d),{ }^{44,46}$ both diffuse and polarization functions $6-31+\mathrm{G}(d)$ and the large $6-311+\mathrm{G}(3 d f){ }^{44,45,47}$

Purely electronic dipole moment, polarizability, and first hyperpolarizability have been computed as first, second, and third energy derivatives of the energy with respect to the field strength, respectively. At the SCF level, all these derivatives have been computed analytically. At the MP2 level, the dipole moment and the polarizability have been computed analytically, whereas the hyperpolarizability has been obtained by numerical differences of the polarizability. At the CI and QCI levels, the dipole moment has been calculated analytically, and the polarizability and the first hyperpolarizability have been computed by single and double numerical differences of dipole moment, respectively. At the MP4 level, both dipole moment and polarizability have been calculated as single and double numerical differences of the energy, respectively. At this level the first hyperpolarizability has not been computed. To consider the effect of triples excitations at the QCI (Ref. 42) level, the dipole moment and the polarizability have also been computed by single and double numerical differences of the energy, respectively. All calculations in this paper have been carried out using the GAUSSIAN-92 (Ref. 48) series of programs.

Derivatives of the purely electronic molecular properties with respect to the nuclear displacements have been found by fitting the dipole moment, the polarizability and the first hyperpolarizability values to a power series in the nuclear coordinate displacements. At the HF and MP2 level, the quadratic force constant has been computed analytically. The MP4, CISD, and QCISD quadratic force constants and all the cubic force constants have been obtained by fitting the energy to a power series in the nuclear coordinate displacements. All the equations are presented in atomic units.

\section{B. Power series expansion of the potential energy}

The potential energy of a diatomic molecule under the effect of an uniform, static electric field is a simultaneous function of both the field strength and the bond length. Then, the energy of such a system can be expressed as a power series expansion,

$$
V(Q, F)=\sum_{n=0} \sum_{m=0} a_{n m} Q^{n} F^{m},
$$

where the first index refers to the nuclei displacements from the equilibrium bond length $Q$, and the second index refers to the strength of the electric field $F$.

Differentiation of Eq. (2), with respect to either nuclear displacements or/and field strength, will lead to relationships between the coefficients $a_{n m}$ of the power series expansion and the potential energy derivatives:

$$
a_{n m}=\frac{1}{n ! m !}\left[\frac{\partial^{(n+m)} V(Q, F)}{\partial Q^{n} F^{m}}\right]_{Q_{e}, F=0} .
$$

Then, the molecular properties are defined:

$$
\begin{aligned}
& \mu_{\mathrm{el}}=-a_{01} ; \alpha_{\mathrm{el}}=-2 a_{02} ; \beta_{\mathrm{el}}=-6 a_{03} ; \\
& k=2 a_{20} ; f=6 a_{30}
\end{aligned}
$$

and their derivatives with respect to either nuclear displacements or/and field strength:

$$
\begin{aligned}
& \left(\frac{\partial \mu_{\mathrm{el}}}{\partial Q}\right)=-a_{11} ;\left(\frac{\partial \alpha_{\mathrm{el}}}{\partial Q}\right)=-2 a_{12} ; \\
& \left(\frac{\partial k}{\partial F}\right)=2 a_{21} ;\left(\frac{\partial^{2} k}{\partial F^{2}}\right)=-\left(\frac{\partial^{2} \alpha_{\mathrm{el}}}{\partial Q^{2}}\right)=4 a_{22} .
\end{aligned}
$$

This paper focuses on a diatomic molecule, so only the parallel component of the field with respect to the dipole moment has been considered. In the power series expansion of the potential energy, the double harmonic approximation including both mechanical and electrical first anharmonic terms has been assumed. In this model, except for the purely mechanical terms, the maximum value for $n$ is 2 , and the maximum value for $m$ is a function of the molecular property of interest in any case. Under these restrictions, the expansion of the double power series of the potential energy used is given by

$$
\begin{aligned}
V(Q, F)= & a_{00}+a_{10} Q+a_{20} Q^{2}+a_{30} Q^{3}+\left(a_{01}+a_{11} Q\right. \\
& \left.+a_{21} Q^{2}\right) F+\left(a_{02}+a_{12} Q+a_{22} Q^{2}\right) F^{2} \\
& +\left(a_{03}+a_{13} Q+a_{23} Q^{2}\right) F^{3}+\cdots .
\end{aligned}
$$

To our knowledge, this is the first systematic study in which the first anharmonic terms are included in the expansion of the potential energy. Lambert ${ }^{14}$ was interested only in relationships between the power series coefficients and both the Stark tuning rate and the IR cross section changes. In the early eighties, Pandey and Santry ${ }^{49}$ applied the mechanical harmonic model to both the potential and vibrational energy expansions. More recently, Castiglioni et al. ${ }^{50}$ applied only the harmonic approximation to the power series expansion of the potential energy. Then, they only found the nuclear re- 
laxation contributions to the electrical properties. Even more recently, Cohen et al. ${ }^{12}$ used different orders of the anharmonic correction for different electrical properties. Inclusion of higher order anharmonic terms in the power series expansion of the potential energy is straightforward following the procedure outlined in this section, and it is summarized in the Appendix.

The nuclear displacements of a molecule caused by an electric field are obtained by differentiation of Eq. (4) with respect to $Q$, and then setting the result equal to zero. Solution of the resulting equation, using a Taylor series expansion, leads to the field-dependent equilibrium coordinate

$$
\begin{aligned}
Q_{\mathrm{eq}}(F)= & -\frac{a_{11}}{2 a_{20}} F \\
& -\left[\frac{a_{12}}{2 a_{20}}-\frac{a_{21}}{a_{20}}\left(\frac{a_{11}}{2 a_{20}}\right)+\frac{3 a_{30}}{2 a_{20}}\left(\frac{a_{11}}{2 a_{20}}\right)^{2}\right] F^{2}+\cdots .
\end{aligned}
$$

The predicted change of the equilibrium geometry induced by the electric field, the so-called nuclear relaxation, ${ }^{50}$ is given by

$$
\begin{aligned}
Q_{\mathrm{nr}} & =\frac{d Q_{\mathrm{eq}}(F)}{d F} \\
& =-\frac{a_{11}}{2 a_{20}}-\left[\frac{a_{12}}{a_{20}}-\frac{2 a_{21}}{a_{20}}\left(\frac{a_{11}}{2 a_{20}}\right)+\frac{3 a_{30}}{a_{20}}\left(\frac{a_{11}}{2 a_{20}}\right)^{2}\right] F+\cdots
\end{aligned}
$$

and is mainly a function (as we will show in Sec. III) of the zeroth-order nuclear relaxation term $Q_{\mathrm{nr}}^{*}$ previously defined by Lambert ${ }^{14}$ as

$$
Q_{\mathrm{nr}}^{*}=\frac{a_{11}}{2 a_{20}} .
$$

This definition of the nuclear relaxation gives only the change of the equilibrium geometry, induced by the applied field, with respect to the zero-field equilibrium geometry. ${ }^{14,50}$ Equation (6) show that the ratio between the dipole moment derivative with respect to the coordinate displacements and the quadratic force constant only controls the change of the equilibrium geometry induced by an applied field. This change of the equilibrium geometry would also be induced by an oscillating field. In this case and due to the nature of the applied field, the equilibrium geometry would also oscillate around the zero-field equilibrium geometry. This induced nuclear relaxation is different, in origin, from the vibrational motion of a molecule even for nonuniform applied fields. Then, these two effects, nuclear relaxation (either constant or time-depending) and vibrational motion of a molecule will be responsible of two different contributions to the molecular electrical properties.

Substitution of Eq. (5) into Eq. (4) will lead to a fielddependent potential energy evaluated at the equilibrium geometry, which will include the effect of the relaxation of the nuclei,

$$
\begin{aligned}
V_{\mathrm{eq}}(F)= & a_{00}+a_{01} F+\left(a_{02}-\frac{a_{11}^{2}}{4 a_{20}}\right) F^{2}+\left[a_{03}-a_{12} \frac{a_{11}}{2 a_{20}}\right. \\
& \left.+a_{21}\left(\frac{a_{11}}{2 a_{20}}\right)^{2}-a_{30}\left(\frac{a_{11}}{2 a_{20}}\right)^{3}\right] F^{3}+\cdots
\end{aligned}
$$

Comparison of this equation and the Taylor series [Eq. (1)] and subtraction of the purely electronic contributions leads to the definition of the nuclear relaxation contributions to the dipole moment $\left(\mu_{\mathrm{nr}}\right)$, polarizability $\left(\alpha_{\mathrm{nr}}\right)$, first hyperpolarizability $\left(\beta_{\mathrm{nr}}\right)$, etc. This definition of the nuclear relaxation contributions to the molecular properties shows their additive character. At the equilibrium geometry and for the zero-field case, the $P_{\mathrm{nr}}$ contributions to the electrical properties are given by

$$
\begin{aligned}
\mu_{\mathrm{nr}} & =0, \\
\alpha_{\mathrm{nr}} & =\frac{a_{11}^{2}}{2 a_{20}}=a_{11} Q_{\mathrm{nr}}^{*}, \\
\beta_{\mathrm{nr}} & =3\left(\frac{a_{12} a_{11}}{a_{20}}-\frac{a_{21} a_{11}^{2}}{2 a_{20}^{2}}+\frac{a_{30} a_{11}^{3}}{4 a_{20}^{3}}\right) \\
& =6\left[a_{12} Q_{\mathrm{nr}}^{*}-a_{21}\left(Q_{\mathrm{nr}}^{*}\right)^{2}+a_{30}\left(Q_{\mathrm{nr}}^{*}\right)^{3}\right] .
\end{aligned}
$$

As Castiglioni et al. ${ }^{50}$ pointed out recently, the nuclear relaxation contributions to the electrical properties are due to the change of the equilibrium geometry induced by the field. In agreement with the recent work of Castilglioni et al. ${ }^{50}$ the harmonic part of these nuclear contributions is a function of two variables, the zeroth-order nuclear relaxation $Q_{\mathrm{nr}}^{*}$, and the derivative of the purely electrical property of the previous order in the field with respect to the coordinate (the $a_{1 m-1}$ coefficient of the power series). As it can be seen, $\mu_{\mathrm{nr}}$ and $\alpha_{\mathrm{nr}}$ are only function of harmonic terms. This is the origin of the zero value for $\mu_{\mathrm{nr}}$, at the equilibrium geometry in absence of a field $\left(a_{10}=0\right)$. The anharmonic part of the $\beta_{\mathrm{nr}}$ is a function of the anharmonic coefficients of the power series expansion of the potential energy and the zeroth-order nuclear relaxation. A similar result has been obtained previously by perturbation theory. ${ }^{17,52}$ The nuclear relaxation contribution to the polarizability and to the first and second hyperpolarizabilities have been shown to be important. ${ }^{8-10,14,50}$ Dykstra et al. ${ }^{25}$ Pandrey and Santry, ${ }^{49}$ Rinaldi et al. ${ }^{51}$ Bishop and Kirtman, ${ }^{52}$ Champagne et al. ${ }^{53}$ and Bartlett et $a l .{ }^{54}$ reach the same conclusion to what they called vibrational contribution to the molecular properties. This amount was essentially due to the induced change of the equilibrium geometry. The $P_{\mathrm{nr}}$ contribution is originated by the nuclei displacement from the zero-field equilibrium geometry, induced by the applied field. In this work, we only have considered uniform fields, but a similar contribution should be obtained for time-oscillating fields. For these fields, the $P_{\mathrm{nr}}$ contribution could be easily coupled with the $P_{\text {vib }}$ contribution, because both effects nuclear relaxation and vibrational motion are time-dependent, but at different frequencies. The 
nuclear relaxation frequency is given by the applied field, and the vibrational motion is given by the vibrational frequencies.

To obtain the vibrational contribution of the molecular properties, derivation of the vibrational energy with respect to the field strength to the corresponding order must be done. In the harmonic model for the vibrational energy, and assuming the zero-point energy as the total vibrational energy (no temperature effect is considered), the $P_{\text {vib }}$ contributions in atomic units are given by

$$
\begin{aligned}
\mu_{\mathrm{vib}} \cong \mu_{\mathrm{ZPE}} & =-\frac{1}{2}\left(\frac{d \omega}{d F}\right)_{Q_{e}, F=0}=-\frac{1}{4(m k)^{1 / 2}}\left(\frac{d k}{d F}\right), \\
\alpha_{\mathrm{vib}} \cong \alpha_{\mathrm{ZPE}} & =-\frac{1}{2}\left(\frac{d^{2} \omega}{d F^{2}}\right)_{Q_{e}, F=0} \\
& =-\frac{1}{4(m k)^{1 / 2}}\left[\left(\frac{d^{2} k}{d F^{2}}\right)-\frac{1}{2 k}\left(\frac{d k}{d F}\right)^{2}\right],
\end{aligned}
$$

$$
\begin{aligned}
\beta_{\mathrm{vib}} \cong \beta_{\mathrm{ZPE}}= & -\frac{1}{2}\left(\frac{d^{3} \omega}{d F^{3}}\right)_{Q_{e}, F=0} \\
= & -\frac{1}{4(m k)^{1 / 2}}\left[\left(\frac{d^{3} k}{d F^{3}}\right)\right. \\
& \left.-\frac{3}{2 k}\left(\frac{d^{2} k}{d F^{2}}\right)\left(\frac{d k}{d F}\right)+\frac{3}{(2 k)^{2}}\left(\frac{d k}{d F}\right)^{3}\right],
\end{aligned}
$$

where $m$ is the reduced mass, $k$ is the quadratic force constant, and $\omega=2 \pi \nu$, where $\nu$ is the vibrational frequency. All such derivatives of the force constant are evaluated at the equilibrium geometry and at zero-field strength. The fielddependent force constant is obtained by double differentiation of the power series [Eq. (4)] with respect to the nuclear displacements,

$$
k_{\mathrm{eq}}(F)=2 a_{20}+6 a_{30} Q_{\mathrm{eq}}+2 a_{21} F+2 a_{22} F^{2}+2 a_{23} F^{3} .
$$

At this point, differentiation of the field dependent force constant with respect to the field strength should be done. Final expressions for the vibrational contributions are given by

$$
\begin{aligned}
\mu_{\mathrm{vib}}= & -\frac{1}{4(m k)^{1 / 2}}\left(2 a_{21}-3 a_{30} \frac{a_{11}}{a_{20}}\right), \\
\alpha_{\mathrm{vib}}= & -\frac{1}{4(m k)^{1 / 2}}\left[4 a_{22}-\frac{a_{21}^{2}}{a_{20}}\right. \\
& \left.-6 a_{30}\left(\frac{a_{12}}{a_{20}}-\frac{a_{21} a_{11}}{2 a_{20}^{2}}+\frac{3 a_{30} a_{11}^{2}}{8 a_{20}^{3}}\right)\right],
\end{aligned}
$$

$$
\begin{aligned}
\beta_{\mathrm{vib}}= & -\frac{1}{4(m k)^{1 / 2}}\left[12 a_{23}-6 \frac{a_{22} a_{21}}{a_{20}}+\frac{3 a_{21}^{3}}{2 a_{20}^{2}}\right. \\
& -9 a_{30}\left(\frac{2 a_{13}}{a_{20}}-\frac{a_{22} a_{11}+a_{21} a_{12}}{a_{20}^{2}}\right. \\
& +3 \frac{a_{21}^{2} a_{11}+2 a_{30} a_{11} a_{12}}{4 a_{20}^{3}}-9 \frac{a_{30} a_{21} a_{11}^{2}}{8 a_{20}^{4}} \\
& \left.\left.+9 \frac{a_{30}^{2} a_{11}^{3}}{16 a_{20}^{5}}\right)\right] .
\end{aligned}
$$

From Eq. (11), the vibrational contributions of the molecular properties are function of derivatives of the force constant with respect to the field strength $\left(a_{21}, a_{22}\right.$, and $a_{23}$ coefficients of the power series expansion). In the double harmonic model of the power series expansion of the potential energy, these coefficients are not included. In consequence, the vibrational contributions to the electrical properties have a null value. Only when the anharmonic terms $\left(a_{30}\right.$ and $a_{2 m}$ coefficients) are included in the power series expansion of the potential energy, the vibrational contributions to the $\mu, \alpha$, and $\beta$ are obtained. Simultaneously, the mechanical anharmonicity is coupled with derivatives of the electrical properties $\left(a_{1 m}\right.$ and $a_{2 m}$ terms). This point has been previously observed for the $\alpha_{\mathrm{vib}}$ contribution for Cohen et al. ${ }^{12}$ and Bishop et al. ${ }^{52}$

Inclusion of the higher order anharmonic terms in the power series expansion leads to more complex expressions of the $P_{\text {vib }}$ contribution to the molecular properties (see the Appendix). While $\mu_{\text {vib }}$ is still unchanged, both $\alpha_{\text {vib }}$ and $\beta_{\text {vib }}$ are corrected by the second-order anharmonic terms $a_{40}$, $a_{31}, a_{32}$.

The experimental values of VSE and VIE given by the Stark tuning rate $\delta_{\nu E}$ and the infrared cross section $\delta_{S E}$, respectively, can also be expressed in terms of the coefficients of the power series. From their definitions and using atomic units, we have

$\delta_{\nu E}=\left(\frac{d \nu}{d F}\right)_{Q_{e}, F=0}=\frac{1}{4 \pi(m k)^{1 / 2}}\left(2 a_{21}-6 a_{30} Q_{\mathrm{nr}}^{*}\right)$

and

$$
\begin{aligned}
\delta_{S E} & =\left(\frac{d \ln I}{d F}\right)_{Q_{e}, F=0} \\
& =\frac{2}{q_{0}}\left(\frac{d q}{d F}\right)_{Q_{e}, F=0}=2\left(\frac{2 a_{12}}{a_{11}}-\frac{a_{21}}{a_{20}}\right),
\end{aligned}
$$

where $m$ is the reduced mass and $q$ is the derivative of the field-dependent dipole moment with respect to the coordinate displacement. Finally, $d q / d F$ is the second derivative of the field-dependent dipole moment with respect to both nuclear displacement and field strength. Both $q$ and $d q / d F$ are evaluated at the equilibrium geometry for the zero-field case.

From Eqs. (11a) and (12a), one can see that $\mu_{\text {vib }}$ is directly related to the Stark tuning rate. In fact, the ratio between the vibrational contribution and the Stark tuning rate is 
TABLE I. Equilibrium bond length (in $\AA$ ) of the $\mathrm{CO}$ molecule at the different levels of theory. The experimental value is $1.128 \AA$ (Ref. 28).

\begin{tabular}{cccccc}
\hline \hline$r_{e}(\AA)$ & $3-21 \mathrm{G}$ & $6-31+\mathrm{G}$ & $6-31 \mathrm{G}(d)$ & $6-31+\mathrm{G}(d)$ & $\begin{array}{c}6-311+\mathrm{G} \\
(3 d f)\end{array}$ \\
\hline RHF & 1.1289 & 1.1295 & 1.1138 & 1.1133 & 1.1026 \\
MP2 & 1.1712 & 1.1760 & 1.1502 & 1.1504 & 1.1334 \\
MP4 & 1.1872 & 1.1939 & 1.1580 & 1.1584 & 1.1406 \\
CISD & 1.1535 & 1.1565 & 1.1357 & 1.1352 & 1.1173 \\
QCISD & 1.1628 & 1.1672 & 1.1446 & 1.1443 & 1.1264 \\
\hline \hline
\end{tabular}

a constant factor. The vibrational contribution of the polarizability can be obtained from what we called in a previous work, ${ }^{9}$ the second order Stark tuning rate. In the same sense, the third and fourth order Stark tuning rates will give the vibrational contributions to the first and second hyperpolarizabilities. The Stark tuning rate is a well known experimental data for some molecules, but only first order Stark tuning rates have been reported until now. ${ }^{13-16}$

At this point, it is important to remark that the $P_{\mathrm{nr}}$ and $P_{\text {vib }}$ contributions to the molecular electrical properties cannot be directly compared with the vibrational contributions arising from perturbation theory methods. This classical notation gives also two different terms for the vibrational contributions. One of them, which is closer to the vibrational term, can be extracted from the zero point vibrational average over the property (ZPVA). The second, known as purely vibrational, is related to the nuclear relaxation term. However, the sum of these terms must have the same value for the two methods. The analysis of the molecular electrical properties presented in this work and the perturbative treatment are compared in detail in Ref. 17.

\section{RESULTS AND DISCUSSION}

In this section the molecular properties and their derivatives computed at different levels of theory are presented and compared with available numerical Hartree-Fock ${ }^{18,19}$ results and experimental data. ${ }^{28-38}$ First, we focus on the equilibrium bond length and the molecular properties calculated; second, we present the molecular properties derivatives obtained; third, calculation of the nuclear relaxation and vibrational contributions of the electrical properties, first order Stark tuning rate, and infrared cross section are presented; finally, a general discussion is given.

In the present study, all the magnitudes presented have been computed at the equilibrium bond length of the $\mathrm{CO}$ molecule at each level of theory used. Unless it is specially specified, the numerical values of these magnitudes for the carbon monoxide are given in atomic units.

In Table I, the calculated bond length of the $\mathrm{CO}$ molecule is presented. With respect to the experimental bond length, the HF level tends to underestimate its value when polarization functions are included in the basis sets. This behavior shows a clear cancellation of errors for the 3-21G and $6-31+\mathrm{G}$ basis sets. Inclusion of the correlation energy tends to give larger values than the experimental bond length, except if a high level of theory and a large basis set is used. Inclusion of triples excitation at the QCISD(T)/6-31
TABLE II. Quadratic force constant $\left[k=\left(\partial^{2} E / \partial r^{2}\right)=2 a_{20}\right]$ of the CO molecule at the different levels of theory. Experimental values are 19.0168 $\mathrm{mdyn} / \AA ̊ \Omega=1.2216$ a.u. (Ref. 28) and $18.55 \mathrm{mdyn} / \AA=1.1915$ a.u. (Ref. 29). (b) Cubic force constant $\left[f=\left(\partial^{3} E / \partial r^{3}\right)=6 a_{30}\right]$ of the CO molecule at the different levels of theory.

\begin{tabular}{lrrrrr}
\hline \hline & & & & & $6-311+\mathrm{G}$ \\
& $3-21 \mathrm{G}$ & $6-31+\mathrm{G}$ & $6-31 \mathrm{G}(d)$ & $6-31+\mathrm{G}(d)$ & $(3 d f)$ \\
\hline (a) $\partial^{2} E / \partial r^{2}$ & & & & & \\
RHF & 1.391 & 1.354 & 1.544 & 1.535 & 1.543 \\
MP2 & 0.979 & 0.950 & 1.171 & 1.158 & 1.186 \\
MP4 & 0.756 & 0.710 & 0.962 & 1.036 & 1.073 \\
CISD & 1.160 & 1.131 & 1.344 & 1.339 & 1.369 \\
QCISD & 1.057 & 1.002 & 1.228 & 1.207 & 1.262 \\
(b) $\partial^{3} E / \partial r^{3}$ & & & & & \\
RHF & -5.18 & -5.14 & -5.44 & -5.74 & -5.85 \\
MP2 & -3.80 & -3.64 & -4.22 & -4.53 & -4.54 \\
MP4 & -3.71 & -3.94 & -5.56 & -4.54 & -4.31 \\
CISD & -4.07 & -4.21 & -4.72 & -4.79 & -4.95 \\
QCISD & -3.82 & -3.95 & -4.38 & -4.53 & -4.74 \\
\hline \hline
\end{tabular}

$+\mathrm{G}(d)$ and QCISD(T)/6-311+ $\mathrm{G}(3 d f)$ levels of theory gave bond length of 1.1480 and $1.1310 \AA$, respectively. Except for the HF/3-21G value, only the use of a very flexible basis set like 6-311+G(3df) and accurate inclusion of the correlation energy allows one to reproduce the experimental bond length (relative error lesser than a 1\%). The dependence of the equilibrium bond length of the $\mathrm{CO}$ molecule with the level of theory used will affect the theoretical determination of the electrical properties. MP4 predicts poor bond lengths considering that both triples and quadruples excitations are included in this wave function.

The quadratic and cubic force constants of the $\mathrm{CO}$ molecule are presented in Tables II(a) and II(b), respectively. With respect to the average of the experimental harmonic force constants (1.217 a.u.) the SCF values are clearly overestimated, and inclusion of the correlation energy tend to decrease the calculated force constant. For the polarized basis sets, while the MP2 and MP4 values are clearly underestimated, the CISD values are still overestimated and the calculated QCISD force constant are also overestimated except for the $6-31+\mathrm{G}(d)$ basis set. The best calculated values are the QCISD with the 6-31G $(d)$ and 6-31+ $\mathrm{G}(d)$ basis sets, that show relative errors lesser than $1 \%$ with respect to the averaged experimental value. On the other hand, the cubic force constant, at least for the correlated levels and the two larger basis sets, shows relative errors smaller than 5\% with respect to the QCISD/6-311+G(3df) value. In general, these errors are smaller than those obtained for the quadratic force constant.

Tables III(a), III(b), and III(c) present the values calculated of the purely electronic component of the electrical properties. As it is well known, the dipole moment of the $\mathrm{CO}$ molecule is a very sensitive property due to its small absolute value. Direct comparison between the SCF dipole moment presented in Table III(a) and the numerical HF (HF/ num) value cannot be done because the numerical $\mu$ has been computed at the experimental bond length. At the experimental geometry, the calculated $\mathrm{HF} / 6-31 \mathrm{G}(d), \mathrm{HF} / 6-31$ $+\mathrm{G}(d)$, and $\mathrm{HF} / 6-311+\mathrm{G}(3 d f)$ values of the dipole mo- 
TABLE III. (a) Electronic component of the dipole moment $\left[\mu_{\mathrm{el}}=-(\partial E /\right.$ $\left.\partial F)=-a_{01}\right]$ of the CO molecule at the different levels of theory. The numerical Hartree-Fock value is -0.10426 (Refs. 18, 19). Experimental values are 0.048 a.u. (Ref. 30) and 0.044 a.u. (Ref. 31). A positive dipole moment means the polarity $\mathrm{C}^{-} \mathrm{O}^{+}$. For the dipole moment, 1 a.u. $=8.47836 \times 10^{30} \mathrm{C} \mathrm{m}=2.54175 \mathrm{D}$. (b) Electronic component of the polarizability $\left[\alpha_{\mathrm{el}}=-\left(\partial^{2} E / \partial F^{2}\right)=-2 a_{02}\right]$ of the $\mathrm{CO}$ molecule at the different levels of theory. The numerical Hartree-Fock value is 14.45 (Refs. 18 and 19). The experimental value is 17.55 a.u. (Ref. 31 ). For the polarizability, 1 a.u. $=1.64878 \times 10^{-41} \mathrm{C}^{2} \mathrm{~m}^{2} \mathrm{~J}^{-1}$. (c) Electronic component of the first hyperpolarizability $\left[\beta_{\mathrm{el}}=-\left(\partial^{3} E / \partial F^{3}\right)=-6 a_{03}\right]$ of the $\mathrm{CO}$ molecule at the different levels of theory. The numerical Hartree-Fock value is 31.32 (Refs. 18 and 19). For the first hyperpolarizability, 1 a.u. $=3.20636 \times 10^{-53}$ $\mathrm{C}^{3} \mathrm{~m}^{3} \mathrm{~J}^{-2}$.

\begin{tabular}{|c|c|c|c|c|c|}
\hline & $3-21 \mathrm{G}$ & $6-31+\mathrm{G}$ & $6-31 \mathrm{G}+(d)$ & $6-31+\mathrm{G}(d)$ & $\begin{array}{c}6-311+\mathrm{G} \\
(3 d f)\end{array}$ \\
\hline \multicolumn{6}{|l|}{ (a) $\mu_{\mathrm{el}}$} \\
\hline RHF & -0.1562 & -0.2081 & -0.1035 & -0.0972 & -0.0574 \\
\hline MP2 & 0.1204 & 0.0688 & 0.0791 & 0.0767 & 0.1046 \\
\hline MP4 & 0.0501 & -0.0077 & 0.0348 & 0.0366 & 0.0771 \\
\hline CISD & -0.0104 & -0.0746 & -0.0183 & -0.0160 & 0.0235 \\
\hline QCISD & 0.0020 & -0.0542 & -0.0045 & -0.0035 & 0.0364 \\
\hline \multicolumn{6}{|l|}{ (b) $\alpha_{\mathrm{el}}$} \\
\hline RHF & 11.17 & 14.17 & 11.99 & 14.10 & 14.05 \\
\hline MP2 & 12.32 & 17.41 & 13.24 & 16.14 & 15.73 \\
\hline MP4 & 11.96 & 17.11 & 13.11 & 16.04 & 15.73 \\
\hline CISD & 11.15 & 16.49 & 12.72 & 15.28 & 14.84 \\
\hline QCISD & 12.03 & 17.04 & 12.98 & 15.75 & 15.32 \\
\hline \multicolumn{6}{|l|}{ (c) $\beta_{\mathrm{el}}$} \\
\hline RHF & 15.67 & 37.10 & 19.43 & 33.53 & 29.67 \\
\hline MP2 & 5.57 & 30.89 & 13.95 & 31.00 & 27.18 \\
\hline CISD & 9.23 & 33.95 & 16.14 & 31.38 & 26.94 \\
\hline QCISD & 7.51 & 33.90 & 14.74 & 31.08 & 27.18 \\
\hline
\end{tabular}

ment are $-0.1304,-0.1269$, and -0.1063 a.u., respectively. These results clearly show two facts. First, very flexible basis sets must be used to reproduce the numerical SCF dipole moment, and second the experimental bond length only can be reproduced if an accurate introduction of the correlation energy is done. Then, when correlation energy is included, both the equilibrium bond length and the calculated $\mu_{\mathrm{el}}$ are getting close to the experimental values. The QCISD/6-311 $+\mathrm{G}(3 d f)$ dipole moment, presented in Table III(a), underestimates the dipole moment by $20 \%$. When the triples excitations are included at the QCISD(T)/6-311+G(3df) level, the $\mu_{\mathrm{el}}$ is 0.0495 a.u., which represents an overestimation from the experimental value. Clearly, contributions different from the purely electronic one must be considered to reproduce the experimental value of the total dipole moment.

For the polarizability and due to its higher absolute value, the agreement between the calculated values and the experimental data is much better. Comparison between the $\mathrm{HF} /$ num and the $\mathrm{HF} / 6-311+\mathrm{G}(3 d f)$ polarizability gave a $3 \%$ underestimation of the MO-LCAO value, which can be due to the different bond length used in the calculations. Therefore, at the correlated levels a better agreement between the calculated and the experimental $\alpha$ should be expected. Inclusion of the triples excitations at the QCISD(T)/ $6-311+\mathrm{G}(3 d f)$ level gave 15.55 a.u. This value underestimates the experimental data by $11 \%$. Consequently, other
TABLE IV. (a) First derivative of the dipole moment with respect to the bond length $\left[(\partial \mu / \partial r)=-a_{11}\right]$ of the CO molecule at the different levels of theory. The experimental value is $-3.22 \times 10^{-10}$ esu=0.670 a.u. (Refs. 3335). (b) First derivative of the polarizability with respect to the bond length $\left[(\partial \alpha / \partial r)=-2 a_{12}\right]$ of the $\mathrm{CO}$ molecule at the different levels of theory. The experimental value is $(2.98 \pm 0.38) \times 10^{-16} \mathrm{~cm}^{2}=(10.6 \pm 1.4)$ a.u. (Refs. 36 and 37). (c) First derivative of the first hyperpolarizability with respect to the bond length $\left[(\partial \beta / \partial r)=-6 a_{13}\right]$ of the $\mathrm{CO}$ molecule at the different levels of theory.

\begin{tabular}{lrrrrr}
\hline \hline & & & & & $6-311+\mathrm{G}$ \\
& $3-21 \mathrm{G}$ & $6-31+\mathrm{G}$ & $6-31 \mathrm{G}(d)$ & $6-31+\mathrm{G}(d)$ & $(3 d f)$ \\
\hline (a) $\partial \mu / \partial r$ & & & & & \\
RHF & -0.851 & -1.072 & -0.987 & -1.059 & -1.014 \\
MP2 & -0.218 & -0.416 & -0.425 & -0.504 & -0.501 \\
MP4 & -0.228 & -0.347 & -0.431 & -0.489 & -0.498 \\
CISD & -0.577 & -0.808 & -0.727 & -0.804 & -0.788 \\
QCISD & -0.496 & -0.713 & -0.644 & -0.719 & -0.711 \\
& & & & & \\
(b) $\partial \alpha / \partial r$ & & & & & \\
RHF & 8.50 & 10.12 & 9.05 & 9.78 & 9.22 \\
MP2 & 10.37 & 14.04 & 11.28 & 12.93 & 12.00 \\
MP4 & 7.59 & 11.26 & 9.76 & 11.33 & 11.11 \\
CISD & 9.04 & 11.42 & 9.85 & 10.89 & 10.14 \\
QCISD & 8.86 & 11.51 & 9.91 & 11.14 & 10.49 \\
(c) $\partial \beta / \partial r$ & & & & & \\
RHF & 20.07 & 15.54 & 27.35 & 16.37 & 10.65 \\
MP2 & -9.95 & -17.15 & 5.59 & -2.27 & -2.29 \\
CISD & 4.03 & -7.26 & 15.07 & 1.44 & 1.37 \\
QCISD & -1.19 & -16.57 & 8.14 & -2.23 & 1.78 \\
\hline \hline
\end{tabular}

contributions different from the pure electronic are needed to reproduce the experimental value.

From the SCF data presented in Table III(c), it can be observed the dependence of the $\beta$ with respect to the basis set and to the geometry used. For the 6-31+ $\mathrm{G}(d)$ and 6-311 $+\mathrm{G}(3 d f)$ basis sets, all the correlated levels predict values of $\beta$ that agree (less than a $10 \%$ error) with the experimental data $^{32}\left(\beta_{\mathrm{ESHG}}=30.2 \pm 3.2\right.$ a.u. $)$ showing the need to include both diffuse and polarization functions in the theoretical calculations of this nonlinear optical property. In a recent review, Shelton and Rice ${ }^{1}$ have established the third harmonic generation (THG) and the static electric field induced second harmonic generation (ESHG) as the preferred experimental techniques to determine the electronic contribution to the hyperpolarizability.

In Tables IV(a), IV(b), and IV(c), the first derivatives of $\mu_{\mathrm{el}}, \alpha_{\mathrm{el}}$, and $\beta_{\mathrm{el}}$ with respect to the normal coordinate are presented. Contrary to the evaluation of the dipole moment, the calculated values of the dipole moment derivative have the correct $\operatorname{sign}^{28}$ for the different levels of theory used in the present work. While the SCF values overestimated this derivative by more than $50 \%$ with the basis sets including polarization functions, the MP2 and MP4 levels underestimate the dipole moment derivative by more than $20 \%$. Like the SCF levels, the CISD and QCISD levels overestimate their predicted dipole moment derivative, but the relative error is reduced to $6.1 \%$, when the $6-311+\mathrm{G}(3 d f)$ basis set is used. However, due to cancellation error the QCISD/6-31G $(d)$ level reproduces, with an error lesser than 5\%, the experimental value of the dipole moment derivative.

The values of the $\partial \beta / \partial Q$ presented in Table IV(b) have 
TABLE V. (a) Second derivative of the dipole moment with respect to the bond length $\left[\left(\partial^{2} \mu / \partial r^{2}\right)=-2 a_{21}\right]$ of the CO molecule at the different levels of theory. The experimental value is $(0.29 \pm 0.10) \times 10^{-2} \mathrm{esu} / \mathrm{cm}^{2}$ $=(0.032 \pm 0.011)$ a.u. (Refs. 33-35). (b) Second derivative of the polarizability with respect to the bond length $\left[\left(\partial^{2} \alpha / \partial r^{2}\right)=-4 a_{22}\right]$ of the CO molecule at the different levels of theory. (c) Second derivative of the first hyperpolarizability with respect to the bond length $\left[\left(\partial^{2} \beta / \partial r^{2}\right)\right.$ $=-12 a_{23}$ ] of the $\mathrm{CO}$ molecule at the different levels of theory.

\begin{tabular}{lrrrrr}
\hline \hline & & & & & $6-311+\mathrm{G}$ \\
& $3-21 \mathrm{G}$ & $6-31+\mathrm{G}$ & $6-31 \mathrm{G}(d)$ & $6-31+\mathrm{G}(d)$ & \multicolumn{1}{c}{$(3 d f)$} \\
\hline (a) $\partial^{2} \mu / \partial r^{2}$ & & & & & \\
RHF & -0.162 & 0.162 & -0.128 & -0.391 & -0.100 \\
MP2 & 1.470 & 0.524 & 0.292 & 0.684 & 0.392 \\
MP4 & 1.351 & 1.508 & 0.982 & 0.960 & 0.744 \\
CISD & 0.198 & -0.093 & 0.004 & -0.111 & -0.198 \\
QCISD & 0.342 & 0.120 & 0.190 & 0.099 & -0.010 \\
(b) $\partial^{2} \alpha / \partial r^{2}$ & & & & & \\
RHF & 4.08 & 6.48 & 5.73 & 7.88 & 7.59 \\
MP2 & 8.81 & 15.27 & 8.86 & 12.20 & 13.62 \\
MP4 & -0.76 & 8.30 & 1.69 & -1.47 & 7.33 \\
CISD & 4.82 & 7.06 & 6.22 & 7.57 & 7.76 \\
QCISD & 2.77 & 4.62 & 4.76 & 6.19 & 6.80 \\
(c) $\partial \beta^{2} / \partial r^{2}$ & & & & & \\
RHF & -7.9 & 6.3 & 4.1 & 17.8 & 26.1 \\
MP2 & -35.7 & -34.1 & -81.8 & -179.0 & 958.3 \\
CISD & 21.7 & 71.7 & 8.9 & 632.2 & -194.5 \\
QCISD & -29.7 & 27.8 & -37.9 & -1001.7 & -341.7 \\
\hline \hline
\end{tabular}

the correct sign. ${ }^{29}$ The different behavior of the dipole and polarizability first derivatives with respect to the level of theory can be due to the different sensitivity of the dipole moment and the polarizability to the change of the bond length. This effect can be observed experimentally in the different changes produce by the environment (spectra in solution) in the line intensities of the infrared and raman vibrational spectroscopy. ${ }^{29}$ With respect to the experimental data, the calculated SCF values of $\partial \alpha / \partial Q$ are underestimated and the MP2 values are overestimated. The CISD and QCISD calculations give values that are into the margin of error of the experimental data, especially when the larger basis sets are used. The calculated values of the first derivative of the $\beta$ with respect to the nuclear displacements presented in Table IV(c) show two different facts. First, the SCF values are clearly overestimated. Second, both correlation energy and very flexible basis sets must be used to obtain reliable values of the $\partial \beta / \partial Q$. It seems that the first derivative of the $\beta$ probably has a small absolute value.

In Tables $\mathrm{V}(\mathrm{a}), \mathrm{V}(\mathrm{b})$, and $\mathrm{V}(\mathrm{c})$, second derivatives of the $\mu, \alpha$, and $\beta$ with respect to the nuclear displacements are presented. The second derivative of the dipole moment with respect to the normal coordinate, like higher order derivatives of dipole moment, has been assumed to be small. ${ }^{55}$ Except for some MP2 and MP4 calculated values, this is what Table V(a) shows. The HF, CISD or QCISD values are either positive or negative, but small in general. The best calculated value $[\mathrm{QCISD} / 6-311+\mathrm{G}(3 d f)]$ is three times larger than the experimental determination, showing that probably all the calculated $\partial^{2} \mu / \partial Q^{2}$ suffer form numerical instabilities. For the $\alpha$ derivative, except the perturbative values, which are very large (MP2) or change even the sign
TABLE VI. (a) Zero order nuclear relaxation $\left(Q_{\mathrm{nr}}^{*}=a_{11} / 2 a_{20}\right)$ of the CO molecule at the different levels of theory. (b) Nuclear relaxation contribution to the polarizability ( $\alpha_{\mathrm{nr}}=a_{11} Q_{\mathrm{nr}}^{*}$ ) of the CO molecule at the different levels of theory. (c) Nuclear relaxation contribution to the first hyperpolarizability [Eq. 8(c)] of the $\mathrm{CO}$ molecule at the different levels of theory.

\begin{tabular}{|c|c|c|c|c|c|}
\hline & $3-21 G$ & $6-31+G$ & $6-31 \mathrm{G}(d)$ & $6-31+\mathrm{G}(d)$ & $\begin{array}{c}6-311+\mathrm{G} \\
(3 d f)\end{array}$ \\
\hline \multicolumn{6}{|l|}{ (a) $Q_{\mathrm{nr}}^{*}$} \\
\hline RHF & 0.607 & 0.796 & 0.640 & 0.688 & 0.657 \\
\hline MP2 & 0.227 & 0.441 & 0.359 & 0.436 & 0.423 \\
\hline MP4 & 0.302 & 0.488 & 0.448 & 0.472 & 0.464 \\
\hline CISD & 0.463 & 0.715 & 0.541 & 0.605 & 0.575 \\
\hline QCISD & 0.470 & 0.711 & 0.524 & 0.596 & 0.563 \\
\hline \multicolumn{6}{|l|}{ (b) $\alpha_{\mathrm{nr}}$} \\
\hline RHF & 0.52 & 0.85 & 0.63 & 0.73 & 0.67 \\
\hline MP2 & 0.05 & 0.18 & 0.15 & 0.22 & 0.21 \\
\hline MP4 & 0.07 & 0.17 & 0.19 & 0.23 & 0.23 \\
\hline CISD & 0.25 & 0.58 & 0.39 & 0.49 & 0.45 \\
\hline QCISD & 0.23 & 0.51 & 0.34 & 0.43 & 0.40 \\
\hline \multicolumn{6}{|l|}{ (c) $\beta_{\mathrm{nr}}$} \\
\hline RHF & -16.80 & -26.47 & -18.97 & -22.64 & -19.67 \\
\hline MP2 & -6.78 & -18.56 & -12.24 & -16.90 & -15.36 \\
\hline MP4 & -6.62 & -15.89 & -13.02 & -15.89 & -15.41 \\
\hline CISD & -12.83 & -26.17 & -16.72 & -20.95 & -18.64 \\
\hline QCISD & -12.65 & -25.82 & -16.08 & -20.77 & -18.58 \\
\hline
\end{tabular}

from one basis set to another (MP4), the HF, CISD values are overestimated with respect to the QCISD ones. In particular, the most favorable cases $[\mathrm{HF} / 6-311+\mathrm{G}(3 d f)$ and CISD/6-311+G(3df)] show relative errors of $11.6 \%$ and $14.1 \%$ with respect to the QCISD/6-311+ $\mathrm{G}(3 d f)$, respectively. The calculated values of the second derivatives of the $\beta$ show even more erratic behavior than the second derivative of the polarizability. This behavior can be due to numerical instabilities of the calculated $\partial^{2} \beta / \partial Q^{2}$. Then, to obtain accurate values of these derivatives analytical derivatives of the electrical properties are needed.

Table VI(a) presents the zeroth-order nuclear relaxation term defined in Eq. (6a). From the experimental values of the dipole moment derivative $e^{33-35}$ and the harmonic force constant, ${ }^{28,29}$ the experimental estimate of the zeroth-order nuclear relaxation is $(0.555 \pm 0.007)$ a.u. The predicted zeroth-order nuclear relaxation value at the QCISD/6-311 $+\mathrm{G}(3 d f)$ differs by less than $2 \%$ from the experimental value. This great agreement that could be fortuitous could also be due to the consistency in the errors observed for the dipole moment derivative and the quadratic force constant at this level of theory with respect to the experimental values. In general, the CISD and QCISD calculations using basis sets including polarization functions predict the $Q_{\mathrm{nr}}^{*}$ with less than a $10 \%$ error with respect to the experimental one. While the SCF tends to overestimate the zeroth-order nuclear relaxation, the MP2 and MP4 levels underestimate it, essentially because of the error in the dipole moment derivative. In the previous section we assessed that the zeroth-order nuclear relaxation term [Eq. 6(b)] represents the major contribution to the total nuclear relaxation. For instance, for a field strength of 0.01 a.u. the $Q_{\mathrm{nr}}^{*}$ term computed at the SCF, CISD, and QCISD levels gave more than $90 \%$ of the total nuclear relaxation. At the MP2 and MP4 levels, the zeroth- 
TABLE VII. (a) Vibrational contribution to the dipole moment [Eq. (11a)] of the $\mathrm{CO}$ molecule at the different levels of theory. (b) Vibrational contribution to the polarizability [Eq. (11b)] of the CO molecule at the different levels of theory. (c) Vibrational contribution to the first hyperpolarizability [Eq. (11c)] of the CO molecule at the different levels of theory.

\begin{tabular}{cccccc}
\hline \hline & & & & & $6-311+\mathrm{G}$ \\
& $3-21 \mathrm{G}$ & $6-31+\mathrm{G}$ & $6-31 \mathrm{G}(d)$ & $6-31+\mathrm{G}(d)$ & $(3 d f)$ \\
\hline (a) $\mu_{\text {vib }}$ & & & & & \\
RHF & -0.0062 & -0.0076 & -0.0065 & -0.0078 & -0.0071 \\
MP2 & 0.0014 & -0.0025 & -0.0025 & -0.0027 & -0.0031 \\
MP4 & 0.0006 & -0.0011 & -0.0034 & -0.0026 & -0.0027 \\
CISD & -0.0035 & -0.0065 & -0.0049 & -0.0058 & -0.0059 \\
QCISD & -0.0032 & -0.0060 & -0.0043 & -0.0053 & -0.0053 \\
& & & & & \\
(b) $\alpha_{\text {vib }}$ & & & & & \\
RHF & 0.07 & 0.08 & 0.07 & 0.08 & 0.07 \\
MP2 & 0.11 & 0.16 & 0.10 & 0.13 & 0.12 \\
MP4 & 0.09 & 0.18 & 0.13 & 0.10 & 0.11 \\
CISD & 0.07 & 0.10 & 0.08 & 0.09 & 0.08 \\
QCISD & 0.07 & 0.11 & 0.08 & 0.10 & 0.09 \\
(c) $\beta_{\text {vib }}$ & & & & & \\
RHF & -0.28 & -0.29 & -0.29 & -0.27 & -0.18 \\
MP2 & 0.20 & -0.30 & -0.19 & -0.44 & 1.86 \\
CISD & $1.1 \cdot 10^{-3}$ & -0.14 & 0.01 & 1.07 & -0.52 \\
QCISD & -0.13 & -0.30 & -0.11 & -2.21 & -2.56 \\
\hline \hline
\end{tabular}

order contribution represents more than $80 \%$ of the total nuclear relaxation. When only the first field-dependent term of the nuclear relaxation is added to the zeroth-order $\left(Q_{\mathrm{nr}} \approx Q_{\mathrm{nr}}^{*}+a_{12} F / 2 a_{20}\right.$ more than the $98 \%$ of the total nuclear relaxation is obtained, showing that these harmonic terms are the most important ones of the total nuclear relaxation. For this reason, in the evaluation of the fielddependent force constant derivatives, only the harmonic terms of the $Q_{\mathrm{nr}}$ have been considered to obtain the vibrational contributions to the electrical properties.

Tables VI(b) and VI(c) present, respectively, the nuclear relaxation contributions to the polarizability and to the first hyperpolarizability. From the experimental values of the dipole moment derivative and the harmonic force constant, the experimental estimate of the $\alpha_{\mathrm{nr}}$ is $(0.372 \pm 0.005)$ a.u. The predicted QCISD/6-311+G(3df) nuclear relaxation contribution to the polarizability is overestimated by $7.5 \%$ with respect to the experimental value. The SCF, MP2, and MP4 $\alpha_{\mathrm{nr}}$ contribution reproduces, essentially, the behavior of the dipole moment derivative. The best estimated value of $\alpha_{\mathrm{nr}}$ is obtained at the QCISD/6-31G $(d)$ level. The calculated $\beta_{\mathrm{nr}}$ takes negative values that have the same order of magnitude than the electronic component, like Tables III(c) and VI(c) show. For instance at the QCISD/6-311+G(3df) level, the absolute value of the $\beta_{\mathrm{nr}}$ represents $68.4 \%$ of the $\beta_{\mathrm{el}}$. This is a general behavior obtained at the different levels of theory considered. At the HF or MP2 with the $6-31 \mathrm{G}(d)$ basis set, the nuclear relaxation contribution has roughly the same absolute value than the electronic one. At the SCF level, the anharmonic terms represent, in average, $8 \%$ of the total $\beta_{\mathrm{nr}}$. However, at the correlated levels these anharmonic terms represent less than $5 \%$ of the total value of $\beta_{\mathrm{nr}}$. For instance, at the QCISD and MP2 using the 6-311+G(3df) basis set,
TABLE VIII. (a) First order Stark tuning rate [Eq. (12a)] of the CO molecule at the different levels of theory (in $10^{7} \mathrm{~cm}^{-1} / \mathrm{V} \mathrm{cm}^{-1}$ ). The experimental value is $(5.09 \pm 1.00) \times 10^{-7} \mathrm{~cm}^{-1} / \mathrm{V} \mathrm{cm}^{-1}$ (Ref. 14). (b) Infrared cross section changes [Eq. (12b)] of the $\mathrm{CO}$ molecule at the different levels of theory (in $10^{9} \mathrm{~cm} / \mathrm{V}$ ). The experimental value is $(-5.5 \pm 5.8) \times 10^{-9} \mathrm{~cm} / \mathrm{V}$ (Ref. 14).

\begin{tabular}{lrrrrr}
\hline \hline & $3-21 \mathrm{G}$ & $6-31+\mathrm{G}$ & $6-31 \mathrm{G}(d)$ & $6-31+\mathrm{G}(d)$ & $\begin{array}{c}6-311+\mathrm{G} \\
(3 d f)\end{array}$ \\
\hline (a) $10^{7} \cdot \delta_{\nu E}$ & & & & & \\
$\mathrm{RHF}$ & 5.32 & 6.47 & 5.55 & 6.69 & 6.06 \\
MP2 & -1.19 & 2.12 & 2.15 & 2.30 & 2.68 \\
MP4 & -0.54 & 0.94 & 2.93 & 2.22 & 2.31 \\
CISD & 2.99 & 5.56 & 4.20 & 4.98 & 4.97 \\
QCISD & 2.69 & 5.13 & 3.64 & 4.52 & 4.56 \\
(b) $10^{9} \cdot \delta_{S E}$ & & & & & \\
RHF & -3.92 & -3.62 & -3.59 & -3.69 & -3.56 \\
MP2 & -17.85 & -12.88 & -10.22 & -9.74 & -9.17 \\
MP4 & -12.22 & -11.79 & -8.41 & -8.40 & -8.94 \\
CISD & -6.47 & -5.52 & -5.26 & -5.29 & -5.05 \\
QCISD & -6.81 & -6.23 & -5.91 & -5.98 & -5.74 \\
\hline \hline
\end{tabular}

the anharmonic terms represent the $4.6 \%$ and $0.9 \%$ of the total $\beta_{\mathrm{nr}}$, respectively.

In Tables VII(a), VII(b), and VII(c) the vibrational contributions to the electrical properties are presented. Comparison between the $\mu_{\mathrm{el}}$ and the $\mu_{\mathrm{vib}}$ shows that the vibrational component is a meaningful component of the total value of the dipole moment. For instance, using the $6-311+\mathrm{G}(3 d f)$ basis set and in absolute value, the $\mu_{\mathrm{vib}}$ represents $11.7 \%$ of the $\mu_{\mathrm{el}}$, in average. As it is shown in the Appendix, higher order anharmonic corrections will not improve the vibrational contribution to the dipole moment. From Tables VII(b) and VII(c), the vibrational contributions to the $\alpha$ and $\beta$ are less important than the nuclear relaxation one. But, both $\alpha_{\text {vib }}$ and $\beta_{\text {vib }}$ must be considered to obtain accurate values of the total $\alpha$ and $\beta$. Specially the $\beta_{\text {vib }}$ represents more than $10 \%$ of the nuclear relaxation contribution, at the QCISD/6-311 $+\mathrm{G}(3 d f)$ level.

In Tables VIII(a) and VIII(b), the calculated Stark tuning rate and IR cross section changes are given. The calculated HF, CISD, and QCISD values of the $\delta_{\nu E}$ agree with the experimental determination. The MP2 and MP4 are quite different than the experimental $\delta_{v E}$. A similar fact can be observed in the theoretically predicted values of the $\delta_{S E}$. While the CISD and QCISD values are in good agreement with the experimentally observed IR cross section change, the MP2 and MP4 values are larger. The origin of this behavior is also due to the large calculated $a_{21}$ coefficient. The SCF estimated $\delta_{S E}$ is smaller than the experimental value because to the large calculated first derivative of the dipole moment [Table IV(a)].

At this point, a comparison between contributions to the molecular properties obtained in the present work and obtained using the finite field methodology ${ }^{8-10}$ to the previously reported data ${ }^{5}$ can be made. Data reported in Ref. 5 are obtained at the HF/DZP level. Then, the comparison must be done with respect to the HF/6-31G(d) values. At the HF/ DZP level, the $r_{\text {eq }}$, the $k$, the $\mu_{\mathrm{el}}$ and the $(\partial \mu / \partial Q) Q_{\mathrm{eq}, F=0}$ values are $1.117 \AA$, 1.525 a.u., -0.070 a.u., and -1.068 a.u., 
respectively. The larger difference between these values and the HF/6-31G $(d)$ ones is obtained for the dipole moment. From these data, the $Q_{\mathrm{nr}}^{*}$ and the $\alpha_{\mathrm{nr}}$ are, respectively, 0.700 and 0.748 a.u. The nuclear relaxation contributions to the dipole moment, polarizability and first hyperpolarizability obtained by the finite field method are $-0.001,0.74$, and -22.17 a.u., respectively. The nonzero value of the $\mu_{\mathrm{nr}}$ gave the numerical error of the finite field values. Vibrational contributions to the electrical properties obtained by the finite field method are $-0.0077,0.06$, and 0.66 a.u., for the $\mu, \alpha$, and $\beta$, respectively. The finite field values are in the same order of magnitude than the values presented in this work. Except for the $\alpha_{\text {vib }}$, the finite field values are slightly overestimated with respect to the $P_{\mathrm{nr}}$ and $P_{\text {vib }}$ contributions calculated in this work. The origin of this overestimation can be due to the different basis set used. But, a systematic slight overestimation of the nuclear and the vibrational contributions to the molecular properties cannot be disregarded.

\section{CONCLUSIONS}

The present study consists in a detailed interpretation of the nuclear relaxation and vibrational contributions to the dipole moment, polarizability and hyperpolarizability of a diatomic molecule. Carbon monoxide has been chosen as an example. The $P_{\mathrm{nr}}$ and $P_{\mathrm{vib}}$ contributions to the static molecular electrical properties, the $\delta_{\nu E}$ and the $\delta_{S E}$ have been interpreted in terms of the derivatives of the electronic components of the $\mu, \alpha$, and $\beta$. These derivatives have been evaluated at the equilibrium geometry and in the zero-field case. The calculated $P_{\mathrm{el}}, P_{\mathrm{nr}}, P_{\mathrm{vib}}, \delta_{\nu E}$, and $\delta_{S E}$ have also been compared with the available experimental data. Finally, the effect of the basis set and the correlation energy and the truncation in the power series expansion have been considered in the study of these molecular properties of the $\mathrm{CO}$ molecule.

With respect to the level of theory, we have found that both very flexible basis sets and correlation energy must be considered to accurately reproduce the experimental data, when they are available. In general and considering the low computational cost of the MP2 calculations, this level of theory allows to obtain reliable values of the different contributions (electronic, nuclear relaxation, and vibrational) to the total molecular electrical properties. For properties with small absolute value, an accurate inclusion of the triples excitations in the treatment of the correlation energy must be included. Analytical determination of the molecular electrical properties and their derivatives with respect to the nuclear displacements is preferred, especially when they have small absolute values.

Nuclear relaxation and vibrational contributions to the total dipole moment, polarizability, and first hyperpolarizability have been evaluated. The relative weight of these contributions to the total molecular electrical properties is increased with the order of the molecular property. In average and using the $6-311+\mathrm{G}(3 d f)$ basis set for the different levels of theory used, these contributions represent $11.7 \%$, $3.3 \%$, and $69.7 \%$ of the electronic contribution of the $\mu, \alpha$, and $\beta$, respectively. It has been shown that the $\mu_{\mathrm{nr}}$ has a zero value for all the diatomic molecules. While the nuclear contribution is null for the dipole moment, for the polarizability and for the first hyperpolarizability represents $2.6 \%$ and $65.1 \%$ of the electronic contribution. The vibrational contribution of these static electrical properties represents $11.7 \%$, $0.6 \%$, and $4.7 \%$ of the electronic contributions. From these data, it can be concluded that all three contributions must be considered to theoretically reproduce the experimentally determinated static electrical properties. In particular for the dipole moment of the $\mathrm{CO}$ molecule, the best predicted value of the total dipole moment obtained in this work is 0.044 a.u., considering the QCISD(T)/6-311+G(3df) electronic component, and the QCISD/6-311+G(3df) vibrational one. This value of the total dipole moment of the $\mathrm{CO}$ molecule agrees with available experimental data. ${ }^{30,31}$ Assuming for the $\mu_{\mathrm{vib}}$, the same relative error that it has been observed for the $\delta_{\nu E}$, the theoretically predicted total dipole moment is 0.047 a.u. This value of the dipole moment is also in agreement with the experimentally determined value. ${ }^{30,31} \mathrm{~A}$ similar result is obtained using the $\mu_{\mathrm{el}}$ values calculated by Scuseria et al. ${ }^{20}$ at the $\operatorname{CCSD}(\mathrm{T})$ level of theory. Only considering both contributions $\mu_{\mathrm{el}}$ and $\mu_{\mathrm{vib}}$ the experimental dipole moment can be reproduced. For the polarizability, the same conclusion can be assumed. Only when the electronic, the nuclear relaxation, and the vibrational contributions are considered, the relative error of the theoretically calculated total polarizability (16.04 a.u.) is reduced to less than $10 \%$ with respect to the experimental value (17.55 a.u.). ${ }^{31}$ Better estimations of the theoretical values could be obtained by analytical evaluation of the electronic component derivatives of the electrical properties. Actually, the methodology presented in Sec. II B is being extended to polyatomic molecules.

The first order anharmonic terms have been considered in the calculated contributions to the electrical properties and in the Stark tuning rate and IR cross section changes by including these terms in the power series expansion of the potential energy. The first order mechanical anharmonic coefficient $a_{30}$ has been showed to be the most important correction to the harmonic model. This is due to the coupling of this term with derivatives of the dipole moment, polarizability, and hyperpolarizability $\left(a_{11}, a_{12}\right.$, and $\left.a_{13}\right)$.

\section{ACKNOWLEDGMENTS}

We thank the computing center of the "Universitat de Girona" for the cpu. This work has been funded though the DGICYT project No. PB92-0333. One of us (J.L.A.) thanks the "Ministerio de Educación y Ciencia" for financial support. We also like to thank Benoit Champagne for sharing results prior to publication.

\section{APPENDIX}

Inclusion of the second order anharmonic corrections in the power series expansion of the potential energy must be done including the $a_{40}$ and $a_{3 m}$ terms. Under these conditions, the potential energy expansion is given by 


$$
\begin{aligned}
V(Q, F)= & a_{00}+a_{10} Q+a_{20} Q^{2}+a_{30} Q^{3}+a_{40} Q^{4} \\
& +\left(a_{01}+a_{11} Q+a_{21} Q^{2}+a_{31} Q^{3}\right) F \\
& +\left(a_{02}+a_{12} Q+a_{22} Q^{2}+a_{32} Q^{3}\right) F^{2} \\
& +\left(a_{03}+a_{13} Q+a_{23} Q^{2}+a_{33} Q^{3}\right) F^{3}+\cdots .
\end{aligned}
$$

Following Sec. II B, it can be easily shown that the nuclear relaxation contributions of these electrical properties are unchanged by the inclusion of the second anharmonic terms in the power series expansion of the potential energy. Only nuclear relaxation of the second hyperpolarizability $\left(\gamma_{\mathrm{nr}}\right)$ will be corrected by the second order anharmonic terms.

Here, the field dependent force constant is given by

$$
\begin{aligned}
k_{\mathrm{eq}}(F)= & 2 a_{20}+6 a_{30} Q+12 a_{40} Q^{2} \\
& +\left(2 a_{21}+6 a_{31} Q\right) F \\
& +\left(2 a_{22}+6 a_{32} Q\right) F^{2} \\
& +\left(2 a_{23}+6 a_{33} Q\right) F^{3}+\cdots
\end{aligned}
$$

and then, the vibrational contributions to the molecular properties are

$$
\begin{aligned}
\mu_{\mathrm{vib}}= & -\frac{1}{4(m k)^{1 / 2}}\left(2 a_{21}-3 a_{30} \frac{a_{11}}{a_{20}}\right), \\
\alpha_{\mathrm{vib}}= & -\frac{1}{4(m k)^{1 / 2}}\left[4 a_{22}-\frac{a_{21}^{2}}{a_{20}}-6 a_{30}\left(\frac{a_{12}}{a_{20}}-\frac{a_{21} a_{11}}{2 a_{20}^{2}}\right.\right. \\
& \left.\left.+\frac{3 a_{30} a_{11}^{2}}{8 a_{20}^{3}}\right)+6 a_{40} \frac{a_{11}^{2}}{a_{20}^{2}}-6 a_{31} \frac{a_{11}}{a_{20}}\right], \\
\beta_{\mathrm{vib}}= & -\frac{1}{4(m k)^{1 / 2}}\left[12 a_{23}-6 \frac{a_{22} a_{21}}{a_{20}}+\frac{3 a_{21}^{3}}{2 a_{20}^{2}}\right. \\
& -9 a_{30}\left(\frac{2 a_{13}}{a_{20}}-\frac{a_{22} a_{11}+a_{21} a_{12}}{a_{20}^{2}}\right. \\
& +3 \frac{a_{21}^{2} a_{11}+2 a_{30} a_{11} a_{12}+2 a_{31} a_{11}^{2}}{4 a_{20}^{3}} \\
& \left.+9 a_{40}\left(4 \frac{a_{11} a_{12}}{a_{20}^{2}}-\frac{a_{21} a_{11}^{2}}{a_{20}^{3}}\right)\right] . \\
& \left.-9 \frac{3 a_{30} a_{21} a_{11}^{2}+4 a_{40} a_{11}^{3}}{8 a_{20}^{4}}+9 \frac{a_{30}^{2} a_{11}^{3}}{16 a_{20}^{5}}\right) \\
& \left.-\frac{a_{12}}{a_{20}}-\frac{a_{21} a_{11}}{a_{20}^{2}}\right)-18 a_{32} \frac{a_{11}}{a_{20}} \\
& \left.-3 \frac{1}{2}\right)
\end{aligned}
$$

As it has been previously mentioned, the inclusion of the anharmonic corrections does not modify the vibrational contribution to the dipole moment, and in consequence, the Stark tuning rate is also not improved by the second order anharmonic corrections. The vibrational contribution to the polarizability is slightly modified with respect to Eq. (11b), and only two extra terms couple with the $a_{40}$ and $a_{31}$ terms are included. The most important effect of the anharmonic corrections is obtained in the first hyperpolarizability. For this nonlinear optical property, inclusion of the power series coefficients $a_{31}, a_{32}$, and $a_{40}$ lead to more complete expression of the $\beta_{\mathrm{vib}}$. The vibrational contributions to higher order nonlinear optical properties will be even more sensitive to the anharmonic terms. From the vibrational contributions obtained in this Appendix, the second order anharmonic corrections $a_{40}, a_{31}$, and $a_{32}$ are coupled with first order anharmonic terms $\left(a_{30}\right.$ and $\left.a_{21}\right)$ and with the nuclear relaxation terms $\left(a_{11} / a_{20}\right.$ and $\left.a_{12} / a_{20}\right)$

${ }^{1}$ D. P. Shelton and J. E. Rice, Chem. Rev. 94, 3 (1994).

${ }^{2}$ D. R. Kanis, M. A. Ratner, and T. J. Marks, Chem. Rev. 94, 195 (1994).

${ }^{3}$ J. L. Brédas, Science 263, 487 (1994).

${ }^{4}$ M. Duran, J. L. Andrés, A. Lledós, and J. Bertrán, J. Chem. Phys. 90, 328 (1989).

${ }^{5}$ J. L. Andrés, A. Lledós, M. Duran, and J. Bertrán, Chem. Phys. Lett. 153, 82 (1989).

${ }^{6}$ J. L. Andrés, M. Duran, A. Lledós, and J. Bertrán, Chem. Phys. 151, 37 (1991).

${ }^{7}$ J. L. Andrés, J. Martí, M. Duran, A. Lledós, and J. Bertrán, J. Chem. Phys. 95, 3521 (1991).

${ }^{8}$ J. Martí, J. L. Andrés, J. Bertrán, and M. Duran, Mol. Phys. 80, 625 (1993).

9 J. L. Andrés, J. Bertrán, M. Duran, and J. Martí, J. Phys. Chem. 98, 2803 (1994).

${ }^{10}$ J. L. Andrés, J. Bertrán, M. Duran, and J. Martí, Int. J. Quantum Chem. 52, 9 (1994).

${ }^{11}$ G. Alagona, R. Camini, C. Ghio, and J. Tomasi, Theor. Chim. Acta 85, 167 (1993).

${ }^{12}$ M. J. Cohen, A. Willets, R. D. Amos, and N. C. Handy, J. Chem. Phys. 100, 4467 (1994).

${ }^{13}$ D. K. Lambert and R. G. Tobin, Surf. Sci. 232, 149 (1990).

${ }^{14}$ D. K. Lambert, J. Chem. Phys. 94, 6237 (1991).

${ }^{15}$ J. S. Luo, R. G. Tobin, D. K. Lambert, G. B. Fisher, and C. L. DiMaggio, J. Chem. Phys. 99, 1347 (1993).

${ }^{16}$ J. S. Luo, R. G. Tobin, and D. K. Lambert, Chem. Phys. Lett. 204, 445 (1993).

${ }^{17}$ J. Martí and D. M. Bishop, J. Chem. Phys. 99, 3860 (1993).

${ }^{18}$ D. Sundholm, P. Pyykkö, and L. Laaksonen, Mol. Phys. 56, 1411 (1985).

${ }^{19}$ L. Laaksonen, P. Pyykkö, and D. Sundholm, Comput. Phys. Rep. 4, 313 (1986).

${ }^{20}$ G. E. Scuseria, M. D. Miller, F. Jensen, and J. Geertsen, J. Chem. Phys. 94, 6660 (1991).

${ }^{21}$ L. A. Barnes, B. Liu, and R. Lindh, J. Chem. Phys. 98, 3972 (1993).

${ }^{22}$ M. Ernzerhot, C. M. Marian, and S. D. Peyerimhoff, Chem. Phys. Lett. 204, 59 (1993).

${ }^{23}$ C. W. Bauschlicher, Jr., Chem. Phys. Lett. 118, 307 (1985).

${ }^{24}$ K. K. Sunil and K. D. Jordan, Chem. Phys. Lett. 145, 377 (1988).

${ }^{25}$ C. E. Dyskstra, S.-Y. Liu, and D. J. Malik, Adv. Chem. Phys. 75, 37 (1989).

${ }^{26}$ V. Kellö, J. Noga, G. H. F. Dierksen, and A. Sadlej, Chem. Phys. Lett. 152, 387 (1988)

${ }^{27}$ H. J. Werner and W. Meyer, Mol. Phys. 31, 855 (1976).

${ }^{28}$ K. P. Huber and G. Herzberg, Molecular Spectra and Molecular Structure. IV. Constants of Diatomic Molecules (Van Nostrand Rienhold, New York, 1979).

${ }^{29}$ J. M. Hollas, Modern Spectroscopy, 2nd ed. (Wiley, New York, 1992).

${ }^{30}$ J. S. Muenter, J. Mol. Spectrosc. 55, 490 (1975).

${ }^{31}$ E. A. Mason and E. W. McDaniel, Transport Properties of Ions in Gases (Wiley-Interscience, New York, 1988).

32 J. F. Ward and C. K. Miller, Phys. Rev. A 19, 826 (1979).

${ }^{33}$ C. Chackerian, Jr. and R. H. Tipping, J. Mol. Spectrosc. 99, 431 (1983).

${ }^{34}$ J. P. Bouanich, N. Van-Thanh, and I. Rossi, J. Quantum Spectrosc. Radiat. Transfer 30, 9 (1983).

${ }^{35}$ B. Rosenblum, A. H. Nethetcot, Jr., and C. H. Townes, Phys. Rev. 109, 400 (1958).

${ }^{36}$ T. Yoshino and H. J. Bernstein, J. Mol. Spectrosc. 2, 213 (1958).

${ }^{37}$ B. Fontal and T. G. Spiro, Spectrochim. Acta A 33, 507 (1977).

${ }^{38}$ R. Aroca, E. A. Robin, and T. A. Ford, Spectrochim. Acta A 33, 499 (1977).

${ }^{39}$ C. Moller and M. S. Plesset, Phys. Rev. 46, 618 (1934). 
${ }^{40}$ R. Krishnan, M. J. Frisch, and J. A. Pople, J. Chem. Phys. 72, 4244 (1980).

${ }^{41}$ J. A. Pople, R. Seeger, and R. Krishnan, Int. J. Quantum. Chem. Symp. 11, 149 (1977).

${ }^{42}$ J. A. Pople, M. Head-Gordon, and K. Raghavachari, J. Chem. Phys. 87, 5968 (1987).

${ }^{43}$ J. S. Binkley, J. A. Pople, and W. J. Hehre, J. Am. Chem. Soc. 102, 939 (1980).

${ }^{44}$ W. J. Hehre, R. Ditchfield, and J. A. Pople, J. Chem. Phys. 56, 2257 (1972).

${ }^{45}$ T. Clark, J. Chandrasekhar, G. W. Spitznagel, and P. v. R. Schleyer, J. Comp. Chem. 4, 294 (1983).

${ }^{46}$ M. J. Frisch, J. A. Pople, and J. S. Binkley, J. Chem. Phys. 80, 3265 (1984).

${ }^{47}$ R. Krishnan, J. S. Binkley, R. Seeger, and J. A. Pople, J. Chem. Phys. 72, 650 (1980).

${ }^{48}$ M. J. Frisch, G. W. Trucks, M. Head-Gordon, P. M. W. Gill, M. W. Wong, J. B. Foresman, B. G. Johnson, H. B. Schlegel, M. A. Robb, E. S. Re- plogle, R. Gomperts, J. L. Andrés, K. Raghavachari, J. S. Binkley, C. González, R. L. Martin, D. J. Fox, D. J. DeFrees, J. Baker, J. J. P. Stewart, and J. A. Pople, Gaussian 92, Gaussian Inc., Pittsburgh, Pennsylvania, 1992.

${ }^{49}$ P. K. K. Pandey and D. P. Santry, J. Chem. Phys. 73, 2899 (1980).

${ }^{50}$ C. Castiglioni, M. Gussoni, M. Del Zoppo, and G. Zerbi, Solid State Commun. 82, 13 (1992).

${ }^{51}$ D. Rinaldi, M. F. Ruiz-López, M. T. C. Martins Costa, and J. L. Rivail, Chem. Phys. Lett. 128, 177 (1986).

${ }^{52}$ D. M. Bishop and B. Kirtman, J. Chem. Phys. 95, 2646 (1991); 97, 5255 (1992).

${ }^{53}$ B. Champagne, E. A. Perpète, and J.-M. André, J. Chem. Phys. 101, 10796 (1994).

${ }^{54}$ L. Adamowicz and R. J. Bartlett, J. Chem. Phys. 84, 4998 (1986); 86, 7250 (1989).

${ }^{55}$ E. B. Wilson Jr., J. C. Decius, and P. C. Cross, Molecular Vibrations. The Theory of Infrared and Raman Vibrational Spectra (Dover, New York, 1980). 